\title{
An MPSK Millimeter-Wave Point-to-Point Link With Radio Over Fiber Synchronous Baseband Receiver
}

\author{
Weidong Zhang, Sining An, Jianpin An, Member, IEEE, \\ Xiangyuan Bu, Member, IEEE, Zhongxia Simon He, Senior Member, IEEE
}

\begin{abstract}
This paper reveals a millimeter-wave point-to-point radio link of MPSK (QPSK, 8-PSK, and 16-PSK) modulation without the need of an analog to digital converter (ADC). The proposed point-to-point radio link uses the clock and data recovery (CDR) applied in the optical-electrical converter (OEC) to recover the MPSK intermediate frequency (IF) signals frequency offset as well as the radio over fiber (RoF) concept to extend the transmit distance of the central unit and the remote radio unit. The proposed point-to-point synchronous radio link removes the high-speed ADC requirement for the digital-intermediatefrequency-over-fiber (DIFoF) link, which can significantly reduce the complexity and the expense of the remote radio unit compared with the standard RoF link. Simultaneously, we have designed a simple symbol synchronization algorithm to realize the realtime point-to-point radio link on a Field Programmable Gate Array (FPGA). The proposed millimeter-wave radio link has successfully demonstrated 6 Gbps QPSK transmission and can tolerate a frequency offset up to $100 \mathrm{MHz}$.
\end{abstract}

Index Terms-Millimeter-wave, synchronization, IFoF, carrier recovery, communication link.

\section{INTRODUCTION}

A $\mathrm{S}$ the Fifth-Generation (5G) mobile systems gradually come into operation and the Sixth-Generation $(6 \mathrm{G})$ is on the horizon, the number of wireless access nodes and the communication speed increase explosively [1]. Significant network densification, which installs a large number of small cells, is widely recognized to meet the high communication capacity demands [2]. Backhauling (connecting cells with the central network) for small cells is considered the most challenging task for such dense deployment, with millimeterwave $(\mathrm{mmW})$ point-to-point (PtP) link and fiber link being two of the principal solutions [3]. MmW PtP links provide an easier small cell deployment, although the hardware cost and power consumption are higher than those of fiber links [4].

It's essential to have a compact mmW PtP link in order to be installed on the top of the high pole, for example. To keep the mmW PtP link's form factor small, the backhaul system frequently uses the RoF concept to transfer the signal from a compact outdoor remote radio unit (RRU) back to a

(Corresponding author: Zhongxia Simon He.)

W. Zhang,X. Bu, and J. An are with the Beijing Institute of Technology, Beijing 100081, China.

S. An is with the Chalmers University of Technology, SE-41296 Gothenburg, Sweden

Z. S. He is with the Chalmers University of Technology, SE-41296 Gothenburg, Sweden and Sinowave AB, Sweden (e-mail: zhongxia@chalmers.se).

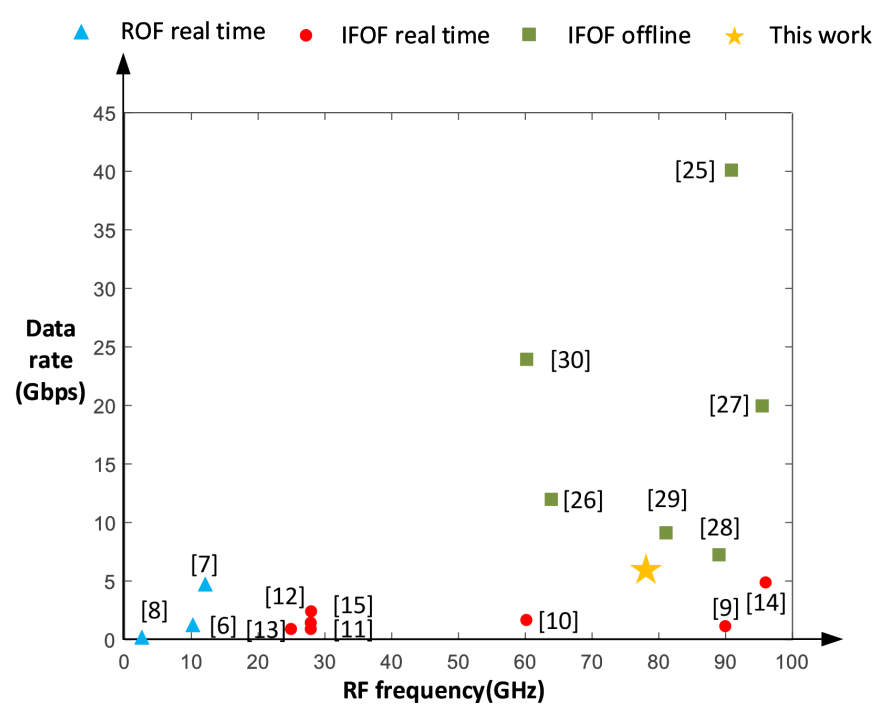

Fig. 1. Overview of the single-channel performance published electrical-based real-time ROF and IFOF wireless system and offline IFOF wireless systems.

bulky, indoor central processing unit (CU), where a powerhungry central processing unit is employed for digitizing and demodulating the transmitted data [5]. Several RoF techniques for signal generation and detection have been proposed for such RRU-CU linkages. Fig. 1 summarizes the current stateof-art real-time RoF link and intermediate frequency over fiber (IFoF) link transmission performance. Modulated signals can be directly generated and received utilizing optical devices (RoF link) at lower radio frequencies, and real-time data transmission (uplink) and receiving (downlink) are revealed, however, only at data rates below 5 Gbps per channel [6]-[8]. At $\mathrm{mmW}$ frequencies, $\mathrm{mmW}$ frontends are often employed to up/down-convert $\mathrm{mmW}$ signals to a lower intermediate frequency and transfer to a CU utilizing the IFoF structure [9][15]. The analog to digital converter (ADC) 's speed usually limits the signal bandwidth of IFoF. However, the transmission data rate apparently can reach 40 Gbps when high sampling rate oscilloscopes are employed in conjunction with offline signal processing. This work proposes a real-time mmW PtP radio link with MPSK modulations for both IFoF architecture uplink and downlink with a data rate of up to $6 \mathrm{Gbps}$.

An IFoF link can be used as an alternative to an RoF link in mm-wave communication systems when the $\mathrm{mmW}$ 
frequencies exceed the cut-off frequency of the cost-effective photoreceiver. There are three main categories of transmission: analog-intermediate-frequency-over-fiber (AIFoF), DIFoF, and sigma-delta-intermediate-frequency-over-fiber (SDIFoF). AIFoF suffers from the non-linearities of the optical link and photoelectric components [6], [7]. To mitigate linearity limitation, the IF signal can be directly digitized by an ADC then transfer to CU as the DIFoF structure, which is employed in the enhanced Common Public Radio Interface (eCPRI) [16]. The bandwidth of the DIFoF link is limited by the sampling rate of Digital-to-Analog converters (DAC) and ADCs.

The One-bit Sigma-Delta modulation (SDM) technique employs a high-speed comparator or optical-electrical converters (OEC) to produce and digitize analog waveforms, which are cost-effective and consume less power than standard ana$\log$ /digital converters [17]. SDM converts a narrowband analog signal into multi-level digital samples by taking advantage of the high sampling rate of comparators or OECs [18]. The SDIFoF link can be used for data transmission using the SDM techniques, with the OEC acting as a DAC to generate modulated signals for transmission. However, the viable analog bandwidth is commonly less than $1 / 10$ of the sampling rate, resulting in a limited bandwidth of the SDIFoF link [19]. In practical communication systems, the asynchronous clock between transceivers will lead to symbol frequency offset (SFO) and carrier frequency offset (CFO). SFO and CFO cancellation is accomplished through differential encoding at the transmitter and differential demodulation at the receiver or by adding a pilot signal (single tone [20], or pseudo-noise (PN) data [21]) at the transmitter and performing signal processing based on such pilot as the reference.

In this paper, we propose an IFoF based mmW PtP synchronization radio link. Unlike previously reported works, the IFoF link in the mmW PtP synchronization system is not only used to lengthen the transmission distance between the RRU and the $\mathrm{CU}$ but also utilized to synchronize received IF signals. The OEC built-in CDR hardware is used to perform pilotless CFO cancellation. And an algorithm is offered for SFO cancellation for real-time data transfer, which is based on the FPGA. The proposed link has been verified in an E-Band (71$76 \mathrm{GHz})$ PtP link test-bed with QPSK, 8-PSK, and 16-PSK modulation. This PtP synchronization radio link realizes a 6 Gbps error-free $\left(\mathrm{BER}<10^{-12}\right)$ transmission in the E-Band test with a frequency offset tolerance up to $\pm 100 \mathrm{MHz}$.

The paper is organized as follows: Section II introduces the suggested $\mathrm{mmW}$ PtP baseband synchronization communication system architecture. In Section III, the principle of the MPSK IF generation and SFO cancellation algorithm is introduced. In Section IV, the experimental setup of the $\mathrm{mmW}$ PtP link test-bed and the experiment results are presented. Finally, in Section V, the paper is summarized .

\section{Proposed PtP mmW Link Structure}

The proposed compact $\mathrm{mmW}$ PtP radio link comprised a CU (IF signal generation and reception) and separated RRUs (for $\mathrm{mmW}$ up and down/conversion). The $\mathrm{CU}$ and the RRU are connected by fibers, which permitted the convenient

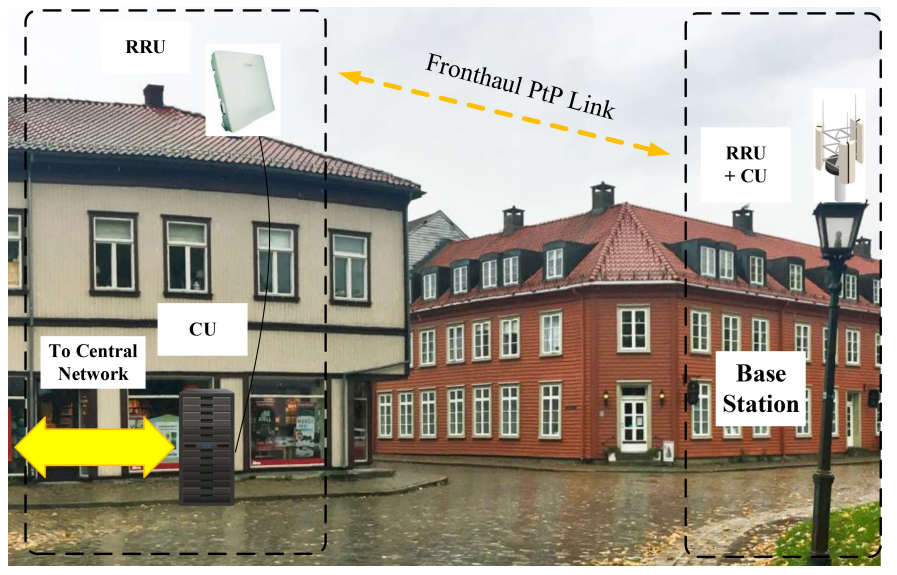

(a)

\section{CU connected with central network}

Central Unit (CU)

Remote Radio Unit (RRU)

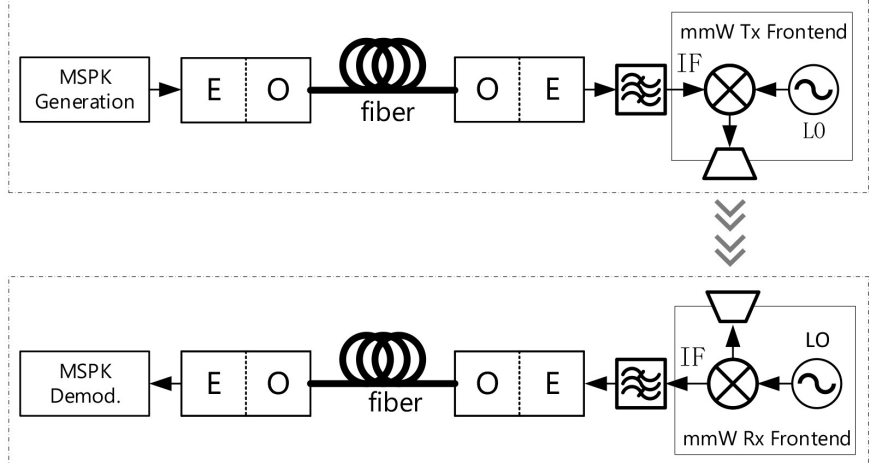

Central Unit (CU)

Remote Radio Unit (RRU)

\section{Base Station}

(b)

Fig. 2. The structure and the application scenarios of the proposed PtP radio link. (a) one scenario of proposed PtP radio link. (b) The system structure of the proposed $\mathrm{mmW}$ PtP radio link.

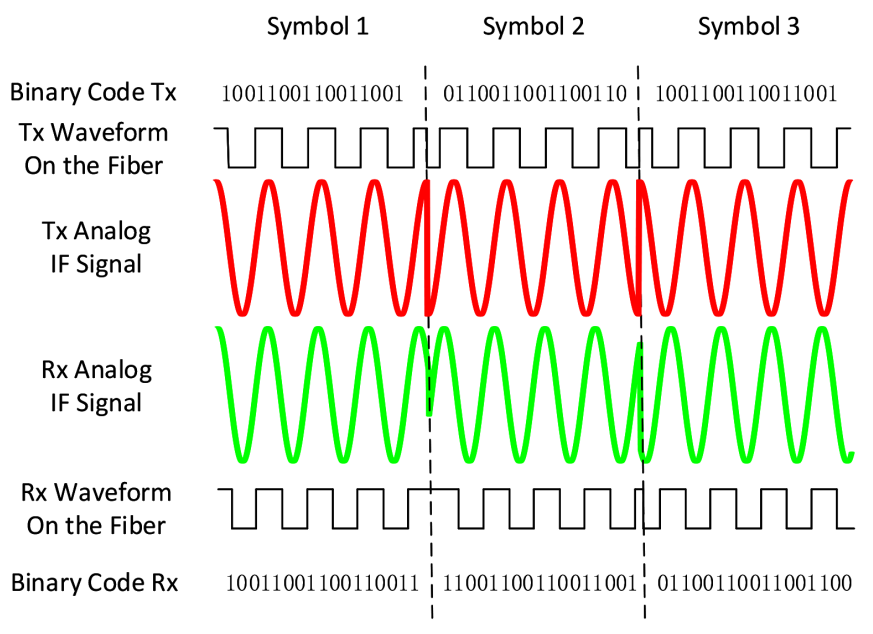

Fig. 3. Waveforms at different nodes that corresponding to BPSK modulated IF signal with carrier frequency offset. Binary codes TX/RX show the data generated/received in the $\mathrm{CU}$. The waveform on the fiber shows the IF binary stream transmitted on the fiber. The $\mathrm{Tx} / \mathrm{Rx}$ analog IF signal is the waveform of the IF signal before/after mmW link transmission. 
deployment of RRUs in the urban scenario. One such scenario is illustrated in Fig. 2a. A small base station is mounted on the street light pole to promote mobile user coverage. However, the fiber network is not available at this installation location. As a result, a fronthaul PtP link is required to connect base station data traffic to the building nearby with fiber network access. The mmW PtP link is either constructed as a compact form (CU and RRU integrated together) on the light pole side or designed as distributed case, as an outdoor RRU, and an indoor CU (as in the building side). This PtP link dualdirectional transmission can be made by frequency division duplex (FDD). In the following discussion, we only focus on the signal directional transmission path for simplicity. For the transmitter path, the user data is generated and modulated in the $\mathrm{CU}$ and transferred as a fixed $10 \mathrm{Gbps}$ binary stream over the fiber and upconvert to $\mathrm{mmW}$ frequency in the RRU. For the receiver path, the received $\mathrm{mmW}$ signal is down-converted by the RRU to the IF signal, where frequency offset occurred owing to asynchronous local oscillators (LO) between the transmitter and the receiver. The down-converted IF signal passes through a low pass filter (LPF) to filter out unwanted high frequency spurious and fed into an OEC. The OEC digitized the IF into a one-bit binary stream and transferred it back to the $\mathrm{CU}$ through the fiber. It should be noted that this OEC has a built-in CDR feature that operates at 10 Gbps. This CDR module assisted the system in performing carrier recovery for the MPSK demodulation, which will be detailed in section III. At the CU side, the received binary stream is then processed by an FPGA in the CU for symbol recovery and demodulation. After the demodulation, the user data is restored, and the bit error rate (BER) and error vector magnitude (EVM) can be calculated. The proposed mmW PtP radio link, with the characteristic of separated RRU and CU, allowed a compact and low-cost RRU design.

\section{PTP SYNCHRONIZATION BASEBAND LINK}

As mentioned in the previous sections, the proposed $\mathrm{mmW}$ PtP radio link comprised neither ADC nor DAC. The analog MPSK IF signal waveform processing is achieved with highspeed binary streams that are transferred through fiber. The principle of such one-bit waveform generation and acquisition is described in detail in this section.

\section{A. MPSK Signal generation}

As illustrated in Fig. 2, the MPSK signal is generated by an FPGA in the CU in the format of the binary stream. Assuming MPSK signal has a symbol rate of $f_{\text {sym }}$, centered at an IF frequency of $f_{I F}$, and the binary stream data rate of $f_{R o F}$. For simplicity, we choose $f_{I F}=k \cdot f_{\text {sym }}$, where $k$ is an integer. For MPSK, there are $2^{M}$ possible modulation points. We choose $f_{R o F}=j \cdot f_{I F}$, where $j \geq 2^{M}$ is also set as an integer. Taking a simple case of BPSK modulated signal as an example, when $f_{I F}=2.5 \mathrm{GHz}, f_{R o F}=10 \mathrm{GHz}$ and $f_{\text {sym }}=$ $0.625 \mathrm{GHz}$. With $j=4$, every 4 bits of binary code can be employed to represent an IF carrier waveform period. As shown in Fig. 3, a 10 Gbps binary code '1001100110011001' generated in the $\mathrm{CU}$ corresponding to a square wave with a

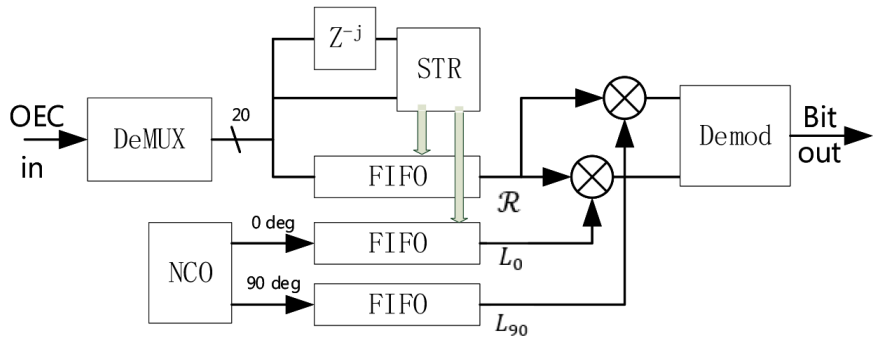

Fig. 4. Signal processing flow diagram of proposed PtP baseband link on the FPGA.

frequency of $2.5 \mathrm{GHz}$. Circular shift the 16-bit code by 2 bits, as '0110011001100110', resulting in a square wave at the same frequency but 180 -degree phase shifted. Notice that in this binary code, every 16-bit can represent a BPSK modulated symbol at $f_{\text {sym }}=0.625 \mathrm{GHz}$. This code is generated in the CU and transferred to the RRU via fiber. Through a bandpass filter (BPF) at the RRU side, the analog IF signal waveform can be obtained for up-conversion to $\mathrm{mmW}$ in the RRU.

\section{B. MPSK Signal Receiving and Recovery}

On the receiver side, the $\mathrm{mmW} \mathrm{Rx}$ frontend in the RRU downconverts the $\mathrm{mmW}$ signal to the received IF signal, as indicated in Fig. 2. It should be noticed that the received IF signal has a different carrier frequency of $f_{I F}+\Delta f$. However, the symbol rate $f_{\text {sym }}$ remains the same as the transmitted signal. The down-converted IF signal is passed through a low pass filter and fed into an OEC, and then converted to the optical signal by the OEC and transmitted through the fiber. In our proposed PtP radio link, a 1310-nm 10GBase-LR Small Form Factor Pluggable (XFP) optical transceiver modules were used as the OEC. A limiting amplifier (LA) is built-in in the OEC to convert the analog waveform into the square wave. The OEC receiver digitized the received IF waveform into the one-bit stream on the $\mathrm{CU}$ side. The OEC receiver is equipped with a CDR function, which will adjust the digitized bitstream rate based on the zero-crossing edges in the received optical waveform [22]. As a result, when the received IF signal is centered at $f_{I F}+\Delta f$, the received binary stream rate is $f_{O E C}=j \cdot\left(f_{I F}+\Delta f\right)$. Note that the received binary stream rate is not integer times of the symbol rate anymore due to the frequency offset $\Delta f$. Therefore a symbol timing recovery (STR) is needed for the correct demodulation.

To elaborate such STR procedure, we assume that the binary stream rate $f_{R o F}=j \cdot f_{I F}=j \cdot k \cdot f_{\text {sym }}$ at the transmitter side. Due to the frequency offset, the received binary stream rate $f_{O E C}=j \cdot\left(f_{I F}+\Delta f\right) \neq l \cdot f_{\text {sym }}$, where the ratio between symbol rate and receiver binary stream as $l=j \cdot k \cdot\left(f_{I F}+\Delta f\right) / f_{I F}$ is not an integer. Given $\Delta f \ll f_{I F}$, we can approximate $l$ as a time-variant integer $l_{n} \in[j k-1, j k, j k+1]$. Assume the received binary stream as $S[n] \in[-1,1]$. The IF carrier waveform is repeats with the continuous phase Within a single transmission symbol. This yields the $n^{t h}$ received symbol $\left(l_{n}+1<n<l_{n+1}\right)$, $S[n]=S[n+j]$. Based on this assumption, we can make STR operation by defining an error function: 


$$
\varepsilon=\sum_{k=l_{n}}^{l_{n+1}-j} S[k] \otimes S[k+j]
$$

STR is made by choosing a $l_{n+1} \in$ $\left[l_{n}+j k-1, l_{n}+j k+1\right]$ that has minimized $\varepsilon$, where ' $\otimes$ ' is a bitwise exclusive-or (XOR) operation.

The signal processing flow diagram of an FPGA implementation in the CU is indicated in Fig. 4. The binary stream received from the OEC is converted into a 20-bit parallel bus with a built-in demultiplexer (DeMUX) to reduce the FPGA operational clock. The data on this parallel bus and its $j$-bit delayed copy is processed by an STR block, with the STR approach described by Eq. 1. The STR block control a first-infirst-out (FIFO) to create a variable length of IF signal vector:

$$
\mathcal{R}=\left[\begin{array}{c}
S\left[l_{n}+1\right] \\
S\left[l_{n}+2\right] \\
\vdots \\
\vdots \\
S\left[l_{n+1}\right]
\end{array}\right]
$$

A numerically controlled oscillator (NCO) generates the carrier waveform of $f_{I F}$ (and it's 90-degree out of phase copy), which are represented by a binary stream at a sampling rate of $f_{O E C}=j \cdot\left(f_{I F}+\Delta f\right)$ and output as 20 bit-width parallel bus. The parallel bus is reshaped by the STR-blockcontrolled FIFOs to give vectors $L_{0}$ and $L_{90}$ that match the size of vector $\mathcal{R}$. The received quadrature baseband signal can be obtained using:

$$
\begin{aligned}
I_{B B}[n] & =\left(\mathcal{R} \otimes L_{0}\right)+\left(\mathcal{R} \otimes L_{0}\right)^{\mathrm{T}} \\
& =\sum_{k=l_{n}+1}^{l_{n+1}} S[k] \otimes L_{0}[k] \\
Q_{B B}[n] & =\left(\mathcal{R} \otimes L_{90}\right)+\left(\mathcal{R} \otimes L_{90}\right)^{\mathrm{T}} \\
& =\sum_{k=l_{n}+1}^{l_{n+1}} S[k] \otimes L_{90} S[k]
\end{aligned}
$$

With demodulated baseband signals $I_{B B}[n]$ and $Q_{B B}[n]$, the received constellation diagram can be monitored, the transmitted bit can be recovered, and the BER performance can be computed.

\section{EXPERIMENTAL RESULTS}

The proposed mmW PtP radio link was tested in two steps: first, an instrument-generated modulated IF signal was used to examine the IFoF synchronization baseband link; second, the proposed PtP synchronous baseband transceiver (CU) was tested with an E-band commercial frontend (RRU), in which the entire single directional transmission mmW PtP radio link was tested. The examination detail and results were presented in the following subsections.

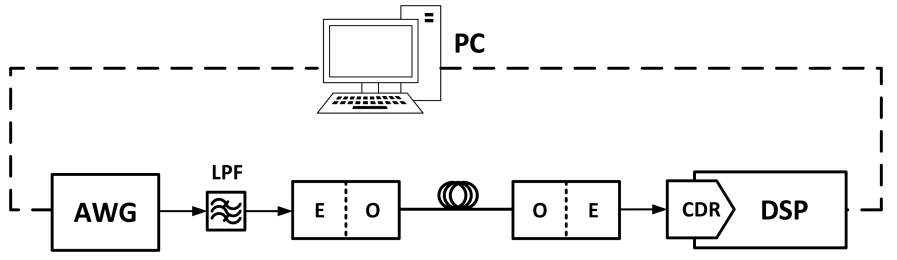

(a)

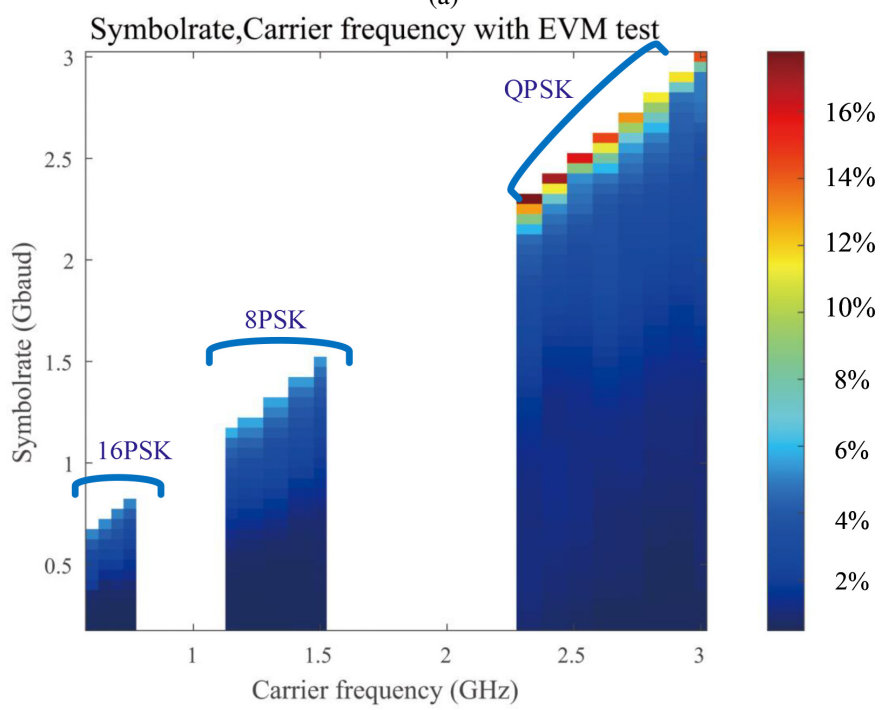

(b)

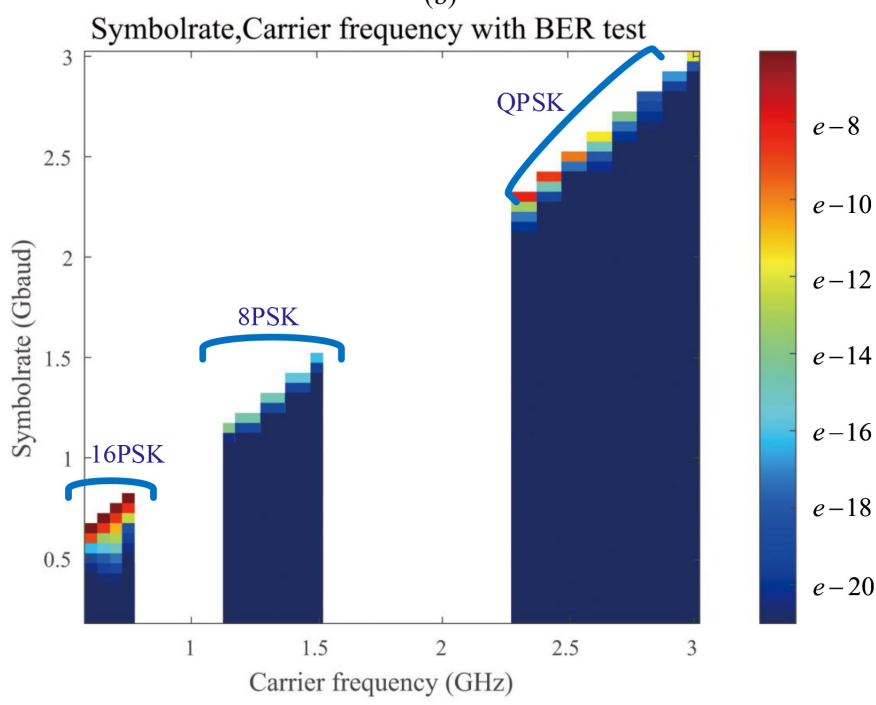

(c)

Fig. 5. mmW PtP synchronization baseband link test set up and test result: (a) experimental setup. (b) received EVM performance with QPSK, 8-PSK and 16-PSK modulations. (c) received BER performance with QPSK, 8-PSK, and 16-PSK modulations.

\section{A. IFoF synchronization baseband link test}

The proposed PtP synchronization baseband transceiver is initially tested with modulated IF signal input. The BER performance, EVM performance, and frequency offset tolerance of the proposed radio link is investigated. The experimental setup is illustrated in Fig. 5a, a modulated IF signal is generated using a 65-Gsps 12-bit Keysight M8195A arbitrary waveform generator (AWG). The IF signal is transmitted through an LPF (cut-off frequency of $8 \mathrm{GHz}$ ), which filters out the unwanted high frequency spurious from the AWG. An 


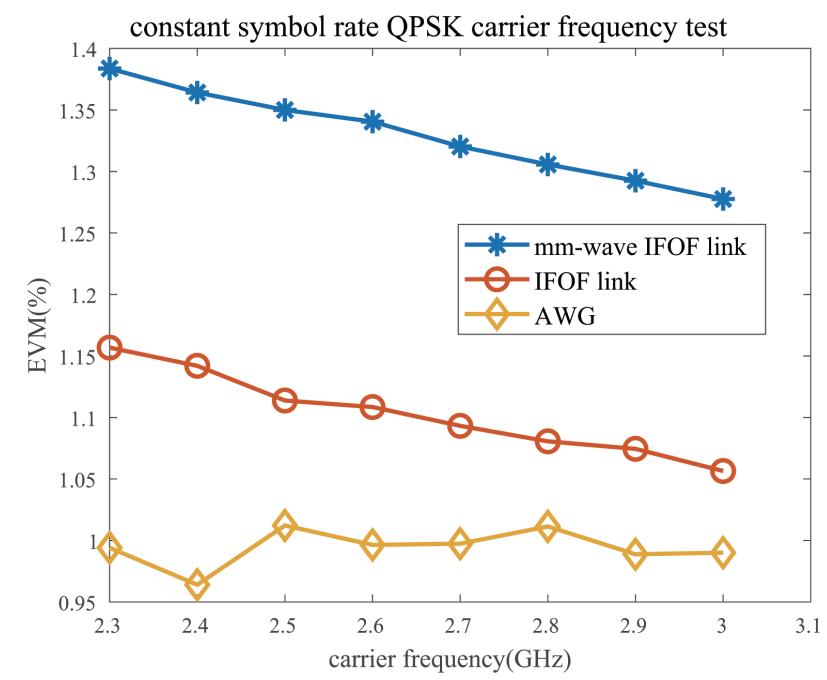

Fig. 6. The EVM performance for the 1.5 GBaud QPSK signal with differente IF carrier frequency.

XFP (Finisar FTLX1413D3BLC) is employed as the OEC in the test, and it converted the MPSK IF signal into the optical signal. The optical signal is transmitted through the fiber. An FPGA (Altera Stratix V GT Evaluation Board) is used at the receiver side as the digital signal processing (DSP) platform. The FPGA platform contains an XFP module of the same type used at the transmitter side. The XFP on the FPGA is equipped with a CDR that adjusts the output data rate of the binary stream. The binary stream is demodulated by the FPGA and then stores in the Random Access Memory (RAM). Then the demodulated data is transferred back to the PC for BER and EVM result analysis.

The AWG generates IF signals with QPSK, 8-PSK, and 16PSK modulations of diverse symbol rates $f_{\text {sym }}$ and different IF frequencies $f_{I F}$; the generated IF signal is then fed into the proposed PtP synchronization baseband link during the test. The test results are as shown in Fig. $5 b-5 c$, the $x-$ axis represents $f_{I F}$, and the y-axis represents $f_{s y m}$. At each point, different colors exhibit the measured EVM for that IF frequencies $f_{I F}$ and symbol rate $f_{s y m}$ combination. From right to left, QPSK, 8-PSK, and 16-PSK modulation results are shown. Note that the IF signal's sampling rate at the receiver is $f_{O E C}$ and must be an integer multiple of the carrier frequency. When there is a frequency offset, the OEC CDR automatically adjusts $f_{O E C}$ to $f_{O E C}=j *\left(f_{I F}+\Delta f\right)$, where $j$ is related to the modulation orders. It can be seen that each modulation has a specific frequency operational $f_{I F}$ range (or lockable range, LR), which can be expressed as $D_{L} / 2^{M}-D_{H} / 2^{M}$, where the $D_{L}$ represents the lowest binary stream bits rate the CDR can be endurance, the $D_{H}$ denotes the highest binary stream bits rate the CDR support, in our case the XFP module supports $8.5 \sim 11.32$ Gbps [23]. In this PtP synchronization baseband link performance test, the QPSK carrier frequency LR is $2.3 \sim 3 \mathrm{GHz}$, the 8-PSK carrier frequency LR is $1.15 \sim 1.5$ $\mathrm{GHz}$, and the 16-PSK carrier frequency LR is $600 \sim 750$ MHz. In addition, we should point out that lower carrier frequencies, i.e., below $1 \mathrm{GHz}, 8-\mathrm{PSK}$, and QPSK, can also be

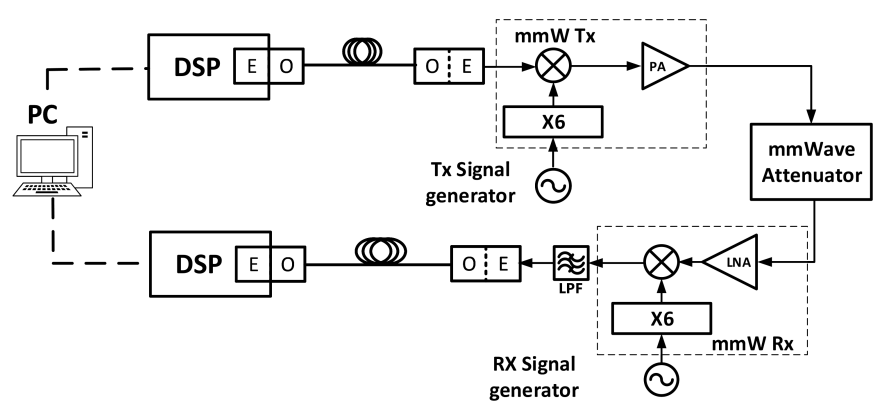

(a)

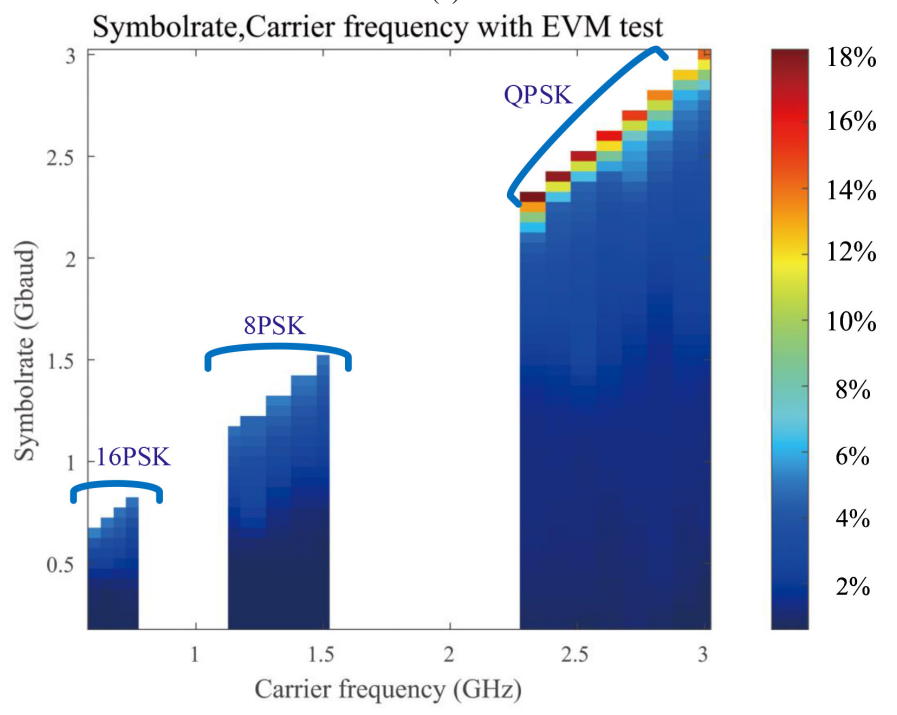

(b)

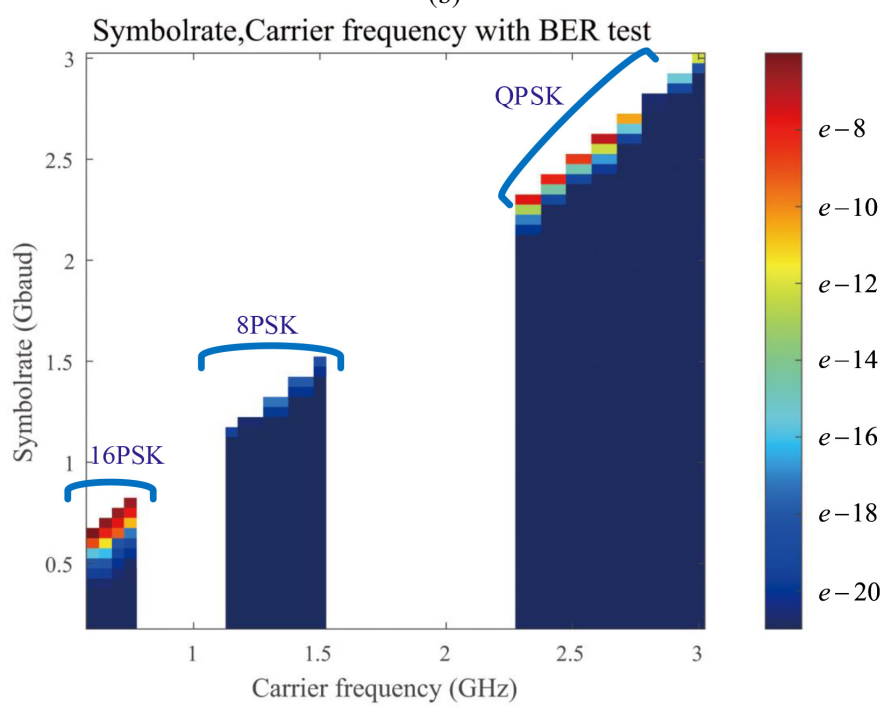

(c)

Fig. 7. mmW PtP radio link test set up and test result: (a) Experimental setup. (b) Received EVM with QPSK, 8-PSK, and 16-PSK modulations. (c) Received BER with QPSK, 8-PSK, and 16-PSK modulations. 

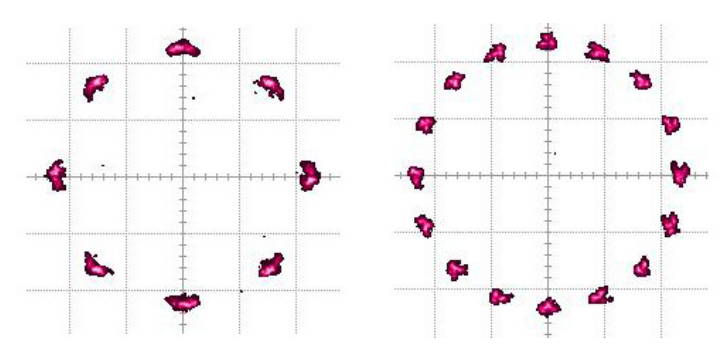

Fig. 8. Received 8-PSK and 16-PSK constellation diagram over asynchronous E-band at different IF frequencies.

demodulated. However, we only presented the highest reliable modulation order for specific carrier frequency/symbol rate combinations in this figure. Since $f_{O E C}$ must be an integer multiple of the carrier frequency $f_{O E C}=j *\left(f_{I F}+\Delta f\right)$, so under variable IF carrier frequencies $f_{I F}$, similar symbol rate $f_{\text {sym }}$ has different oversampling rate (OSR). As demonstrated in Fig. 6, for a constant symbol rate, the EVM performance is improved with an increase in the carrier frequency when the AWG output signal has a constant EVM performance.

It can be observed from the Fig. 5b-5c, that to demodulate 16-PSK, the OEC requires oversampling rate greater than 16 . So the upper limit of $f_{I F}$ is $0.75 \mathrm{GHz}$ with $f_{\text {sym }}$ of 0.6 Gbaud. This scenario also implies less phase error margin for XFP CDR to determine the IF waveform zero-crossing. The best EVM results for $16-\mathrm{PSK}$ is $0.56 \%$ with $f_{I F}=750$ $\mathrm{MHz}, f_{\text {sym }}=200 \mathrm{Mbaud}$. On the other hand, a 6 Gbps QPSK modulated IF signal can be successfully received with an EVM of $14.25 \%$ and a BER of $1.2 \times 10^{-12}$.

The operation of MPSK demodulation depends on the ability of CDR in the XFP to recover the IF signal without any phase error. When the IF signal is upconverted to the $\mathrm{mmW}$ frequency and transmitted through the mmW link, the IF signals receive performance deteriorates with the increase in phase noise. Therefore it is important to repeat a similar test simultaneously with the commercial mmW RRU units.

\section{B. MmW PtP radio link test at E-band}

The proposed PtP synchronization radio link is examined at E-band with commercial $\mathrm{mmW}$ frontends: the Gotmic gTSC0023 at the transmitter (Tx) and the Gotmic gRSC0015 at the receiver $(\mathrm{Rx})$, which are operated at $78 \mathrm{GHz}$. Two signal generators (Agilent E8257D) supply the LO signals to the transmitter and receiver modules separately. An mmW digital controlled attenuator is inserted between Tx and Rx to investigate the dynamic range of the $\mathrm{mmW} \mathrm{PtP}$ synchronization radio link. The IF signal input of the Tx and IF signal output of the Rx is connected to an FPGA at the CU through fibers using OECs, which is described in the previous sections. As a result, the $\mathrm{mmW}$ frontends as RRU can be separately deployed away from the CU.

QPSK, 8-PSK, and 16-PSK modulation signals are transmitted through the proposed $\mathrm{mmW}$ PtP radio link. EVM is processed offline with PC with waveform captured and stored in the FPGA, and the BER is tested in real-time communication with software programmed within the DSP. The measurement results are summarized and plotted in Fig.

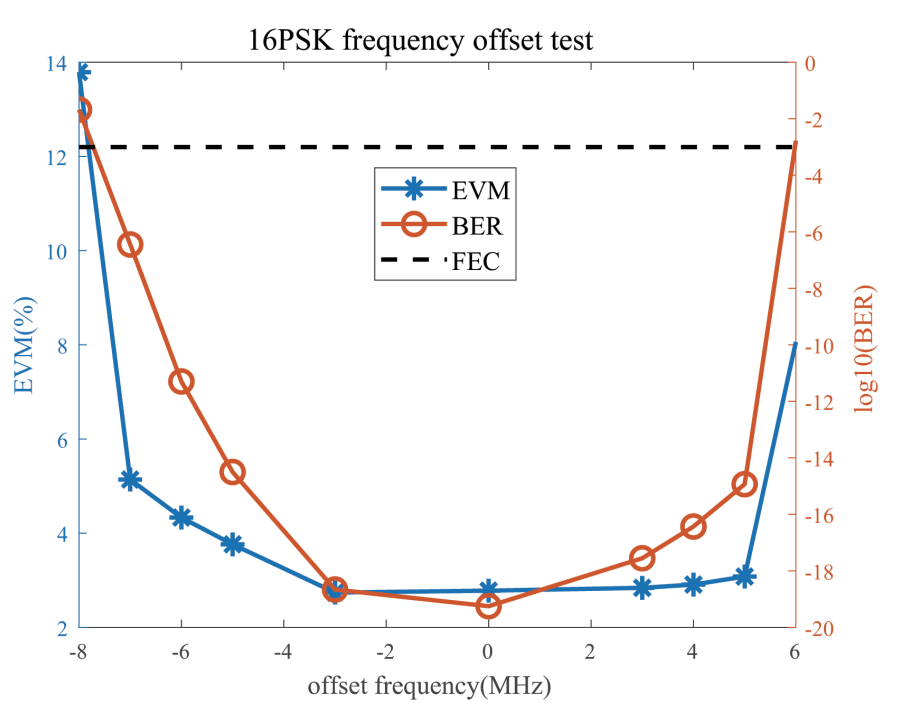

(a)

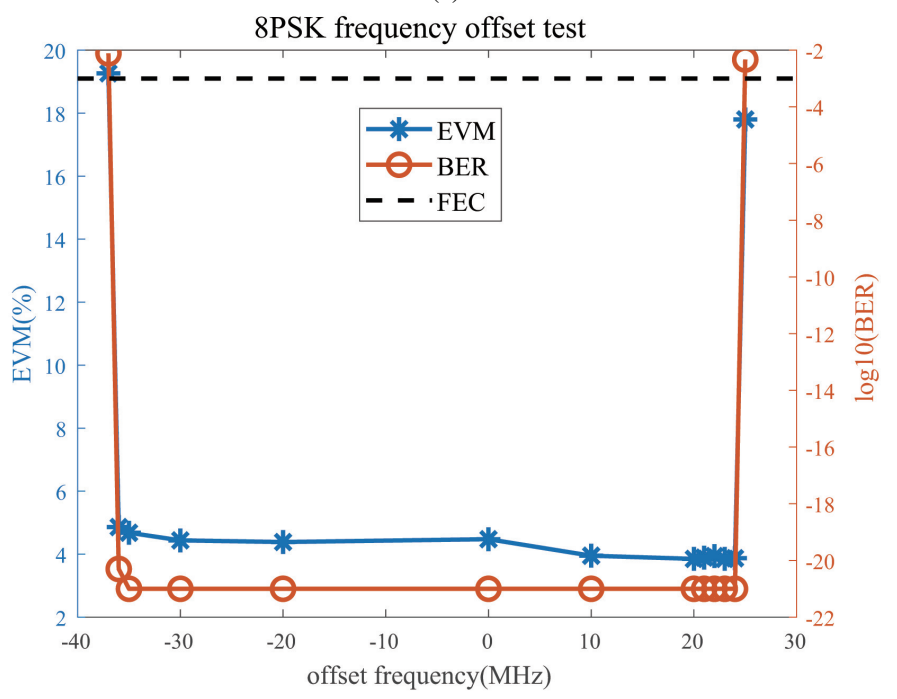

(b)

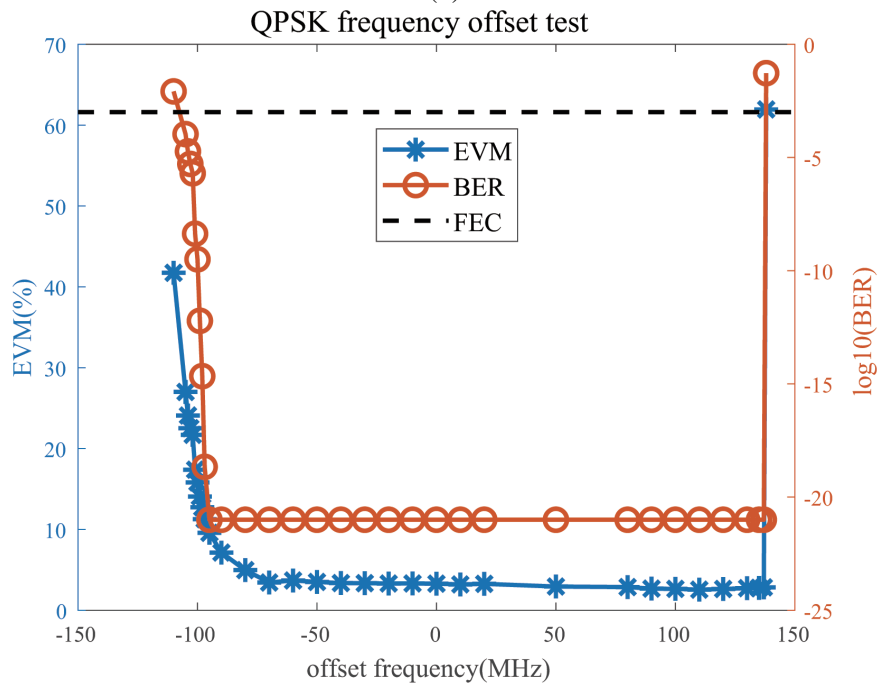

(c)

Fig. 9. The proposed mmW PtP radio link frequency offset tolerance with different modulation types. (a) 16-PSK. (b) 8-PSK. (c) QPSK. 


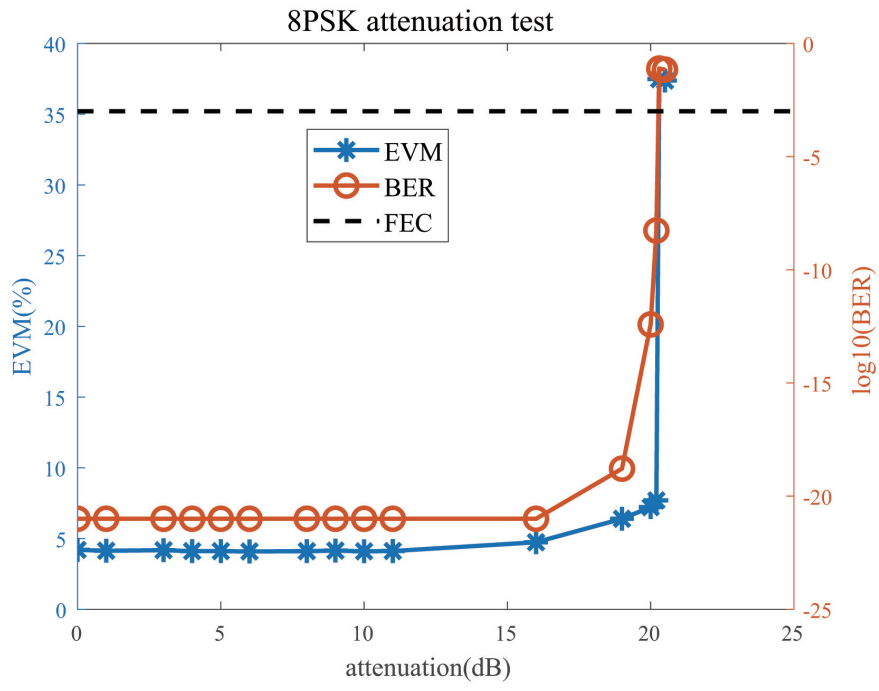

Fig. 10. The measured dynamic range of the mmW PtP radio link with 1 Gbaud 8-PSK transmission at $1.5 \mathrm{GHz}$ IF frequency.

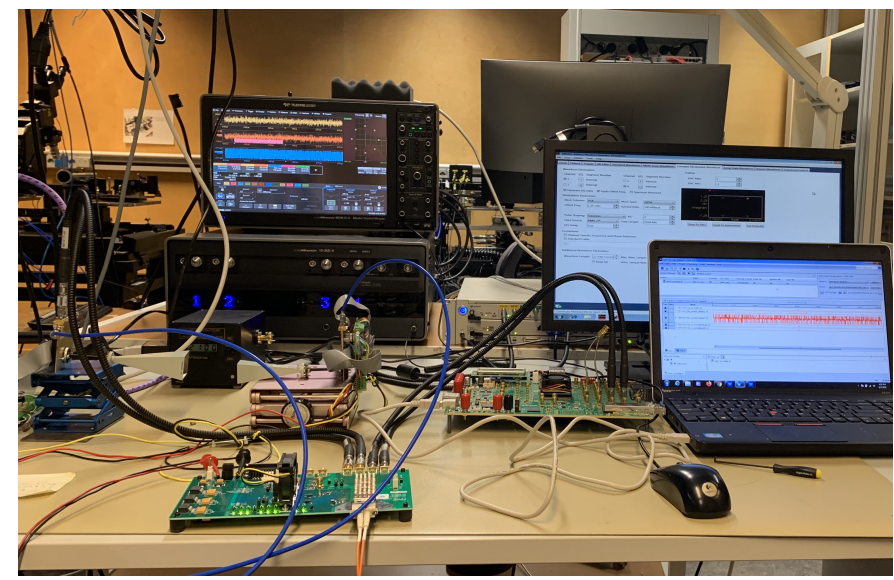

Fig. 11. The test setup of the mmW PtP radio link at the Lab.

7b-7c. It can be observed that the performance is slightly degraded owing to non-linearity, bandwidth limitation, and phase noise introduced by the $\mathrm{mmW}$ frontends. For example, as shown in Fig.7b, the 3 Gbaud QPSK central at $3 \mathrm{GHz}$ $\mathrm{mmW}$ PtP radio link has an EVM of $14.85 \%$ and a BER of $8.39 \times 10^{-12}$ compared with the EVM of $14.25 \%$ and the BER of $1.2 \times 10^{-12}$ in the baseband link test. However, the test result satisfies the 3GPP requirement, in which the bound of the QPSK EVM is $17.5 \%$ [24]. A 1.5 Gbaud 8-PSK transmission with a $1.5 \mathrm{GHz}$ IF frequency exhibits an EVM of $5.59 \%$ and a BER of $1.25 \times 10^{-15}$. Similarly, the EVM of 600 Mbaud 16-PSK transmissions with the IF frequency of $750 \mathrm{MHz}$ is $5.14 \%$, and the BER is $5.68 \times 10^{-9}$.

The receiver LO is deliberately adjusted away from the transmitter frequency to investigate the receiver's frequency offset tolerance. The test result is demonstrated in Fig. 9. For 16-PSK modulation, it needs $f_{O E C}$ to be 16 times higher than $f_{I F}$. Limited by the maximal speed of the OEC $D_{H}$, it left less margin for frequency offset error. Only a $12 \mathrm{MHz}$ frequency offset is allowed for the maintenance of the BER-

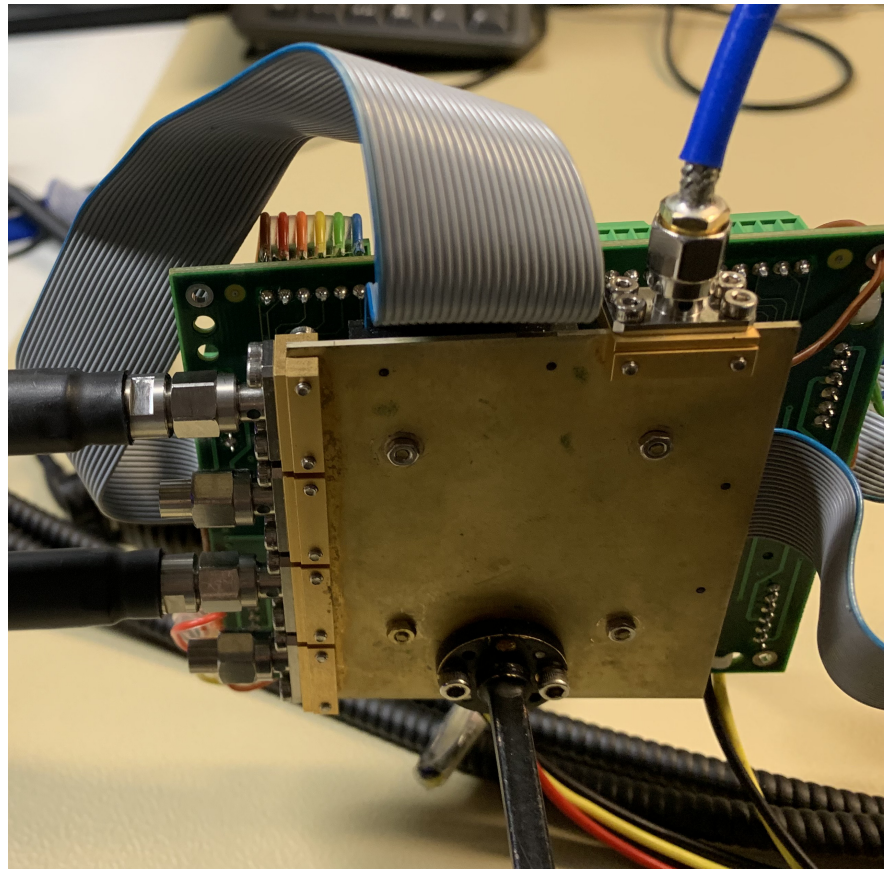

Fig. 12. The commercial Eband module in the mmW PtP radio link test.

free transmission. The frequency offset range of the BER-free transmission of the mmW PtP link can increase to $50 \mathrm{MHz}$ and $160 \mathrm{MHz}$ for the 8-PSK and QPSK transmission cases, respectively. And the measurement indicated that this link has tolerance $\pm 100 \mathrm{MHz}$ frequency offset with the $6 \mathrm{Gbps}$ QPSK transmission. The modulated IF signal experiment exhibits that the proposed PtP radio link can tolerate a broad range of $f_{I F}$ offset and still support error-free data transmission. The test constellation diagrams are shown in Fig. 8 with different modulations.

We also evaluated the dynamic range of the $\mathrm{mmW}$ PtP synchronization radio link. However, it should be noticed that we did not include a variable gain amplifier (VGA) at the IF signal stage. The dynamic range has only exhibited the proposed RoF baseband synchronization link's sensitivity, which can be improved if VGA is added. The result is shown in Fig. 10, that the $21 \mathrm{~dB}$ dynamic range is achieved in a 1 Gbaud 8-PSK transmission at $1.5 \mathrm{GHz}$ carrier frequency.

\section{CONCLUSION AND DisCSSION}

This paper demonstrates an $\mathrm{mmW}$ PtP radio synchronization link. A CDR-based MPSK modulation carrier synchronization is tested based on the mm-wave IFoF transmission architecture. We also proposed an STR algorithm for realtime transmission on the FPGA. The measurement results of the transmission performance of different modulation types, carrier frequencies, and symbol rates have been studied. The test result exhibited that the proposed PtP radio link has successfully transmitted 6 Gbps QPSK transmission over a $78 \mathrm{GHz}$ wireless link with frequency offset $\pm 100 \mathrm{MHz}$, and the received signal has an EVM at $14.85 \%$ and BER at $8.39 \times 10^{-12}$, which satisfies the $3 \mathrm{GPP}$ requirement. Compare with the traditional ROF link design, the proposed PtP radio 
TABLE I

STATE-OF-THE-ART ON EXPERIMENTAL DEMONSTRATIONS FOR MMWAVE ROF LINKS

\begin{tabular}{|c|c|c|c|c|c|c|c|c|c|c|c|}
\hline Ref & $\begin{array}{l}\text { RoF } \\
\text { method }\end{array}$ & $\begin{array}{c}\mathrm{RF} \\
\text { freq. } \\
(\mathrm{GHz})\end{array}$ & $\begin{array}{c}\text { Aggregate } \\
\text { capacity. } \\
\text { (Gbps) }\end{array}$ & $\begin{array}{l}\text { Photodiode } \\
\text { sample-rate. } \\
\text { (GHz) }\end{array}$ & $\begin{array}{l}\text { Single-bandl } \\
\text { data-rate. } \\
\text { (Gbps) }\end{array}$ & $\begin{array}{c}\text { INumber } \\
\text { of } \\
\text { bands }\end{array}$ & $\begin{array}{l}{ }^{2} \text { Uplink } \backslash \\
\text { Downlink }\end{array}$ & Modulation & EVM & $\begin{array}{l}\text { Photo diode } \\
\text { type }\end{array}$ & $\begin{array}{l}\text { Optical wave } \\
\text { length }(\mathrm{nm})\end{array}$ \\
\hline$[8]$ & SDoF+realtime & 2.365 & 0.14 & 10 & 0.14 & 1 & No $\backslash$ Yes & 16-QAM & $1.6 \%$ & SFP & 850 \\
\hline [7] & ARoF+realtime & 12 & 4.8 & 15 & 4.8 & 1 & Yes $\backslash$ No & OFDM $\backslash 16-Q A M$ & $6 \%$ & DML & \\
\hline [13] & SDIFoF+realtime & 25.07 & 1.92 & 40 & 0.96 & 2 & No $\backslash$ Yes & 64-QAM & $6.4 \%$ & QSFP & 850 \\
\hline [15] & AIFoF+realtime & 28 & 1 & 10 & 1 & 1 & Yes $\backslash$ Yes & BPSK & & SFP & \\
\hline [6] & ARoF+realtime & 10.5125 & 2.28 & 15 & 1.14 & 2 & No $\backslash$ Yes & $\mathrm{OFDM} \backslash 16-\mathrm{QAM}$ & $8 \%$ & DML & $1532.69,1536.95$ \\
\hline [9] & AIFoF+realtime & 90 & 1.5 & 90 & 1.5 & 1 & Yes \Yes & multi tone $\backslash \mathrm{DQPSK}$ & $14 \%$ & MZM & $1548.5 \sim 1549.5$ \\
\hline [11] & AIFoF+realtime & 28 & 2 & 6 & 1 & 2 & Yes $\backslash$ Yes & multi tone $\backslash 64-\mathrm{QAM}$ & $7.5 \%$ & DML & $\begin{array}{c}1550,1510,1570, \\
1530,1270,1330\end{array}$ \\
\hline [14] & AIFoF+realtime & 96 & 5 & 50 & 5 & 1 & Yes $\backslash$ Yes & multi tone $\backslash \mathrm{DQPSK}$ & $8 \%$ & MZM & \\
\hline [10] & AIFoF+realtime & 60 & 1.6 & 10 & 1.6 & 1 & No $\backslash$ Yes & $\mathrm{OFDM} \backslash 16-\mathrm{QAM}$ & $10.5 \%$ & MZM & 1270,1578 \\
\hline [12] & AIFoF+realtime & 28 & 9.042 & 6 & 2.5 & 4 & Yes $\backslash$ Yes & multi tone $\backslash 64-\mathrm{QAM}$ & $8 \%$ & DML & $\begin{array}{c}1550,1510,1570, \\
1530,1270,1330\end{array}$ \\
\hline [25] & AIFoF+offline & 91 & 80 & 100 & 40 & 2 & No $\backslash$ Yes & FBMC $\backslash \mathrm{OQAM}$ & $30 \%^{\circ}$ & $\begin{array}{l}\text { tical IQ modulator } \\
\text { +DPMZM }\end{array}$ & \\
\hline [26] & ARoF+offline & 64 & 12 & 10 & 12 & 1 & $\mathrm{No} \backslash$ Yes & multi tone $\backslash \mathrm{QPSK}$ & $13.2 \%$ & EML & 1557.3 \\
\hline [27] & AIFoF+offline & 95 & 20 & 100 & 20 & 1 & $\mathrm{No} \backslash$ Yes & $\mathrm{OFDM} \backslash 16-\mathrm{QAM}$ & $14.6 \%$ or & tical IQ modulator & $1530 \sim 1570$ \\
\hline [28] & AIFoF+offline & 88.9 & 7 & 100 & 7 & 1 & No $\backslash$ Yes & 16-QAM & $5.9 \%$ or & tical IQ modulator & 1550 \\
\hline [29] & AIFoF+offline & 81 & 18 & 100 & 9 & 2 & Yes $\backslash$ Yes & FBMC $\backslash O Q A M$ & $13.8 \%$ & MZM & \\
\hline [30] & AIFoF+offline & 60 & 24 & 10 & 24 & 1 & Yes \Yes & multi tone $\backslash 16$-QAM & $12 \%$ & MZM & 1550 \\
\hline This worl & k AIFoF+realtime & 78 & $6 \backslash 4.5 \backslash 3$ & 12 & $6 \backslash 4.5 \backslash 3$ & 1 & Yes $\backslash$ Yes & QPSK $\backslash 8$-PSK $\backslash 16-P S K$ & K $15 \%$ & XFP & 1310 \\
\hline
\end{tabular}

link does not need the high-speed ADC or high sample rate SDM at the RRU; hence it provides low complexity and low expense for the RRU design. In addition, suppose a higher bit rate OEC is applied in the proposed PtP radio synchronization link, the higher achievable data rate can be attained, which can take the $\mathrm{mmW}$ or sub- $\mathrm{THz}$ communication system's vast bandwidth property better. Overall, we think this $\mathrm{mmW}$ PtP radio synchronization link is one of the essential potential choices for realizing the high bandwidth wireless communication systems due to the low complexity and lowcost property.

This work is also compared with previously reported RoF data transmission as summarized in the Table. I

As shown in Table. I, with ARoF and powerful offline processing, an offset-QAM-based filterbank multicarrier (FBMCOQAM) and $2 \times 2$ multiple-input multiple-output (MIMO) is applied to improve the system capacity, [25], [29], which can achieve 80 Gbps transmission at the $90 \mathrm{GHz}$ RF frequency. There is also IEEE 802.11ad packet modulated at $88.9 \mathrm{GHz}$ succeeded transmitted through the IFoF mmW link [28]. The IFoF link and distributed antenna can also be used in the data transmission on the high-speed train (HTS), in which a switched wavelength-division multiplexing is used to transmit $20 \mathrm{Gbps}$ modulated at W-band [27]. There are some experiments based on multi-tone communication which also demonstrate the 24 Gbps transmission capacity [26], [30]. The state-of-art real-time RoF link and IFoF link transmission performance are concluded in Fig. 1, and Table. I and the offline IFoF link transmission performance is also included in this summary. Owing to the limitation of the high-speed ADC and DAC, the achievable data rate of the real-time IFoF transmission is significantly lower than the offline transmission. The real-time IFoF $\mathrm{mmW}$ link applied in the HTS experiment has achieved a data rate of $1.5 \mathrm{Gbps}$ [9]. A $1.6 \mathrm{Gbps}$ orthogonal frequency-division multiplexing (OFDM) modulation outdoor transmission is successfully real- time transmitted by an applied analog-IFoF link [10]. The IFoF-based distributed antenna system (DAS) with $2 \times 2$ MIMO achieved 2 Gbps transmission in the indoor scenario [11], the same setup with $4 \times 4$ MIMO successfully transmitted around $9 \mathrm{Gbps}$ into the IFoF-based mobile fronthaul [12]. The SDIFoF based on the QSFP-100G in the experiment also demonstrated the 960Mbps transmission [13]. The IFoF link can also be used in the $8 \mathrm{~K}$ videos transmission [14]; in this experiment, the sum-data rate can achieve 5 Gbps. Furthermore, a simple synchronization IFoF link used BPSK to achieve a 1 Gbps data rate [15]. As can be observed from the table, this work demonstrated an ADC-less MPSK real-time wireless transmission solution at $70 / 80 \mathrm{GHz} \mathrm{mmW}$ band.

\section{ACKNOWLEDGMENT}

The work is partially funded by EU H2020 project Int5Gent (957403) and Car2tera 824962 Projects of International Cooperation and Exchanges NSFC (61620106001).

\section{REFERENCES}

[1] K. Miyanabe, T. Gama Rodrigues, Y. Lee, H. Nishiyama, and N. Kato, "An Internet of Things Traffic-Based Power Saving Scheme in CloudRadio Access Network," IEEE Internet of Things Journal, vol. 6, no. 2, pp. 3087-3096, 2019.

[2] C. Wu, H. Li, O. Caytan, J. Van Kerrebrouck, L. Breyne, J. Bauwelinck, P. Demeester, and G. Torfs, "Distributed Multi-User MIMO Transmission Using Real-Time Sigma-Delta-Over-Fiber for Next Generation Fronthaul Interface," Journal of Lightwave Technology, vol. 38, no. 4, pp. 705-713, 2020.

[3] Ajung Kim, Young Hun Joo, and Yungsoo Kim, "60 GHz wireless communication systems with radio-over-fiber links for indoor wireless LANs," IEEE Transactions on Consumer Electronics, vol. 50, no. 2, pp. 517-520, 2004.

[4] I. A. Alimi, A. L. Teixeira, and P. P. Monteiro, "Toward an Efficient C-RAN Optical Fronthaul for the Future Networks: A Tutorial on Technologies, Requirements, Challenges, and Solutions," IEEE Communications Surveys Tutorials, vol. 20, no. 1, pp. 708-769, 2018. 
[5] A. Bekkali, T. Kobayashi, K. Nishimura, N. Shibagaki, K. Kashima, and Y. Sato, "Performance evaluation of real-time $10 \mathrm{GbE}$ data connectivity over a converged IF-over-Fiber links and millimeter-wave wireless bridge," in 2017 IEEE International Conference on Communications (ICC), 2017, pp. 1-6.

[6] M. Chen, X. Xiao, J. Yu, X. Li, and F. Li, "Real-Time Gigabit RS-Coded OFDM Signal Transmission over WDM-Based X-Band $2 \times 2$ MIMO RoF System," in Optical Fiber Communication Conference. Optical Society of America, 2017.

[7] M. Chen, X. Xiao, J. Yu, F. Li, Z. R. Huang, and H. Zhou, "Demonstration of Software-Reconfigurable Real-Time FEC-Enabled 4/16/64 QAM-OFDM Signal Transmission in an X-Band RoF System," IEEE Photonics Journal, vol. 8, no. 2, pp. 1-8, 2016.

[8] I. C. Sezgin, M. Dahlgren, T. Eriksson, M. Coldrey, C. Larsson, J. Gustavsson, and C. Fager, "A Low-Complexity Distributed-MIMO Testbed Based on High-Speed Sigma-Delta-Over-Fiber," IEEE Transactions on Microwave Theory and Techniques, vol. 67, no. 7, pp. 2861-2872, 2019.

[9] A. Kanno, P. T. Dat, N. Yamamoto, T. Kawanishi, N. Iwasawa, N. Iwaki, K. Nakamura, K. Kawasaki, N. Kanada, N. Yonemoto, Y. Sato, M. Fujii, K. Yanatori, N. Shibagaki, and K. Kashima, "High-Speed Railway Communication System Using Linear-Cell-Based Radio-Over-Fiber Network and Its Field Trial in 90-GHz Bands," Journal of Lightwave Technology, vol. 38, no. 1, pp. 112-122, 2020.

[10] K. Kanta, A. Pagano, E. Ruggeri, M. Agus, I. Stratakos, R. Mercinelli, C. Vagionas, P. Toumasis, G. Kalfas, G. Giannoulis, A. Miliou, G. Lentaris, D. Apostolopoulos, N. Pleros, D. Soudris, and H. Avramopoulos, "Analog fiber-wireless downlink transmission of $\mathrm{IFoF} / \mathrm{mm}$ Wave over in-field deployed legacy PON infrastructure for 5G fronthauling," IEEE/OSA Journal of Optical Communications and Networking, vol. 12, no. 10, pp. D57-D65, 2020.

[11] M. Sung, J. Kim, E. Kim, S. Cho, Y. Won, B. Lim, S. Pyun, J. K. Lee, and J. H. Lee, "5G Trial Services Demonstration: IFoF-Based Distributed Antenna System in $28 \mathrm{GHz}$ Millimeter-Wave Supporting Gigabit Mobile Services," Journal of Lightwave Technology, vol. 37, no. 14, pp. 3592-3601, 2019.

[12] M. Sung, J. Kim, E. Kim, S. Cho, Y. Won, B. Lim, S. Pyun, H. Lee, J. K. Lee, and J. H. Lee, "RoF-Based Radio Access Network for 5G Mobile Communication Systems in $28 \mathrm{GHz}$ Millimeter-Wave," Journal of Lightwave Technology, vol. 38, no. 2, pp. 409-420, 2020.

[13] C. Wu, H. Li, J. Van Kerrebrouck, A. Vandierendonck, I. L. de Paula, L. Breyne, O. Caytan, S. Lemey, H. Rogier, J. Bauwelinck, P. Demeester, and G. Torfs, "Distributed Antenna System Using SigmaDelta Intermediate-Frequency-Over-Fiber for Frequency Bands Above $24 \mathrm{GHz}$," Journal of Lightwave Technology, vol. 38, no. 10, pp. 27652773, 2020.

[14] A. Bekkali, T. Kobayashi, K. Nishimura, N. Shibagaki, K. Kashima, and Y. Sato, "Millimeter-Wave-Based Fiber-Wireless Bridge System for $8 \mathrm{~K}$ UHD Video Streaming and Gigabit Ethernet Data Connectivity," Journal of Lightwave Technology, vol. 36, no. 18, pp. 3988-3998, 2018.

[15] C. Liu, A. Liu, B. Wu, and H. Yin, "Design and implementation of IFoF high-speed full-duplex system based on $28 \mathrm{GHz}$ base-station," in 2017 IEEE 9th International Conference on Communication Software and Networks (ICCSN), 2017, pp. 1-4.

[16] "Common public radio interface: eCPRI interface specification V2.0," May 2019. [Online]. Available: http://www.cpri.info/spec.html

[17] T. Pfeiffer, "Next Generation Mobile Fronthaul and Midhaul Architectures," J. Opt. Commun. Netw., vol. 7, no. 11, pp. B38-B45, Nov 2015.

[18] G. Chang, L. Cheng, M. Xu, and D. Guidotti, "Integrated fiber-wireless access architecture for mobile backhaul and fronthaul in $5 \mathrm{G}$ wireless data networks," in 2014 IEEE Avionics, Fiber-Optics and Photonics Technology Conference (AVFOP), 2014, pp. 49-50.

[19] S. Cho, H. S. Chung, C. Han, S. Lee, and Jong Hyun Lee, "Experimental demonstrations of next generation cost-effective mobile fronthaul with IFoF technique," in 2015 Optical Fiber Communications Conference and Exhibition (OFC), 2015, pp. 1-3.

[20] S. An, Z. S. He, J. Chen, H. Han, J. An, and H. Zirath, "A Synchronous Baseband Receiver for High-Data-Rate Millimeter-Wave Communication Systems," IEEE Microwave and Wireless Components Letters, vol. 29, no. 6, pp. 412-414, 2019.

[21] S. An, Z. S. He, J. Li, X. Bu, and H. Zirath, "Coded Pilot Assisted Baseband Receiver for High Data Rate Millimeter-Wave Communications," IEEE Transactions on Microwave Theory and Techniques, vol. 68, no. 11 , pp. 4719-4727, 2020

[22] V. A. Thomas, M. El-Hajjar, and L. Hanzo, "Millimeter-Wave Radio Over Fiber Optical Upconversion Techniques Relying on Link Nonlinearity," IEEE Communications Surveys Tutorials, vol. 18, no. 1, pp. 29-53, 2016.
[23] finisar, "Industrial Temperature 10Gb/s 10km XFP Optical Transceiver FTLX1413M3BTL."

[24] 3GPP, "Technical Specification Group Radio Access Network; NR ; Base Station (BS) radio transmission and reception (Release 17) ," Technical Specification (TS), Jan 2021, version 17.0.0.

[25] P. T. Dat, A. Kanno, F. Rottenberg, J. Louveaux, N. Yamamoto, and T. Kawanishi, " $80 \mathrm{~Gb} / \mathrm{s} 2 \times 2$ MIMO Fiber-Wireless Integrated System in W Band Using IFoF Transmission," in 2019 International Topical Meeting on Microwave Photonics (MWP), 2019, pp. 1-4.

[26] C. Vagionas, S. Papaioannou, N. Argyris, K. Kanta, N. Iliadis, G. Giannoulis, D. Apostolopoulos, H. Avramopoulos, C. Caillaud, H. Debregeas, G. Kalfas, and N. Pleros, "A 6-Band $12 \mathrm{~Gb} / \mathrm{s} \mathrm{IFoF/V-Band} \mathrm{Fiber-}$ Wireless Fronthaul Link Using an InP Externally Modulated Laser," in 2018 European Conference on Optical Communication (ECOC), 2018, pp. 1-3.

[27] P. T. Dat, A. Kanno, K. Inagaki, F. Rottenberg, N. Yamamoto, and T. Kawanishi, "High-Speed and Uninterrupted Communication for HighSpeed Trains by Ultrafast WDM Fiber-Wireless Backhaul System," Journal of Lightwave Technology, vol. 37, no. 1, pp. 205-217, 2019.

[28] K. Fujiwara, A. Kanno, H. Yamaoka, and K. Tokita, "IEEE802.11ad Packet Transmission on IF-over-Fiber in the W-Band with SSB Optical Modulation," in 2019 European Microwave Conference in Central Europe (EuMCE), 2019, pp. 618-621.

[29] F. Rottenberg, P. T. Dat, T. Nguyen, A. Kanno, F. Horlin, J. Louveaux, and N. Yamamoto, " $2 \times 2$ MIMO FBMC-OQAM Signal Transmission Over a Seamless Fiber-Wireless System in the W-band," IEEE Photonics Journal, vol. 10, no. 2, pp. 1-14, 2018.

[30] N. Argyris, G. Giannoulis, K. Kanta, N. Iliadis, C. Vagionas, S. Papaioannou, G. Kalfas, D. Apostolopoulos, C. Caillaud, H. Debrégeas, N. Pleros, and H. Avramopoulos, "A 5G mmWave Fiber-Wireless IFoF Analog Mobile Fronthaul Link With up to 24-Gb/s Multiband Wireless Capacity," Journal of Lightwave Technology, vol. 37, no. 12, pp. 28832891, 2019

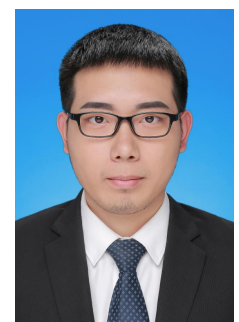

Weidong Zhang received the B.S. degree in communication and electronics engineering from the Beijing Institute of Technology, Beijing, China, in 2016. He is currently a Ph.D. student with the School of Information and Electronics, Beijing Institute of Technology.

His research interests include millimeter wave communication, IFoF based C-RAN system, the MIMO precoding in frequency selective channel and RIS aided cell-free network.

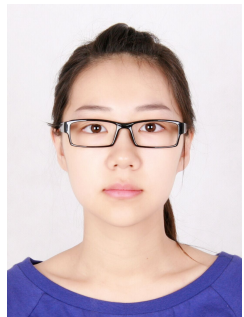

Sining An received the B.S. and M.Sc. degrees in communication and electronics engineering from the Beijing Institute of Technology, Beijing, China, in 2013 and 2016, respectively. She is currently pursuing the Ph.D. degree with the Microwave Electronics Laboratory, Department of Microtechnology and Nanoscience, Chalmers University of Technology. Her research interests include high data rate communication, modulation and demodulation, and high-resolution radar. 


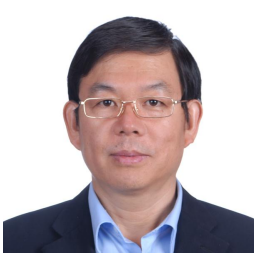

Jianping An (Member, IEEE) received the Ph.D. degree from the Beijing Institute of Technology, China, in 1996. He joined the School of Information and Electronics, Beijing Institute of Technology, in 1995, where he is currently a Full Professor. He is currently the Dean of the School of Information and Electronics, Beijing Institute of Technology. His research interests are in the fields of digital signal processing, cognitive radio, wireless networks, and high-dynamic broadband wireless transmission technology.

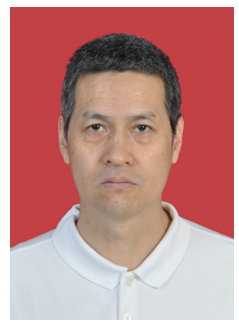

Xiangyuan Bu (Member, IEEE) received the B.Eng.and Ph.D. degrees from the Beijing Institute of Technology (BIT), Beijing, China, in 1987 and 2007, respectively. From 1996 to 1998, he was a Visiting Scholar at the Military Academy of Belarus, Minsk, Belarus. Since 2002, he has been with the School of Information and Electronics, BIT, where he is currently a Professor. His current research interests include wireless communication, digital signal processing, and channel coding.

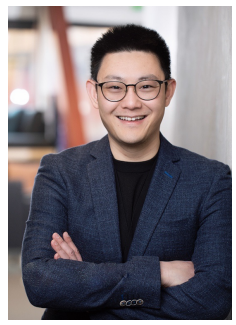

Zhongxia Simon He (Senior Member, IEEE) received the M.Sc. degree from the Beijing Institute of Technology, Beijing, China, and the Ph.D. degree from the Chalmers University of Technology, Gothenburg, Sweden, in 2008 and 2014, respectively.

He is currently an Associate Professor with the Microwave Electronics Laboratory, Department of Microtechnology and Nanoscience (MC2), Chalmers University. His current research interests include high data rate wireless communication, modulation and demodulation, mixed-signal integrated circuit design, radar, and packaging. He is also jointly working at SinoWave AB, Sweden. 